\title{
Evidence of breakdown of the spin symmetry in diluted 2D electron gases
}

\author{
P. Giudici, ${ }^{1}$ A. R. Goñi ${ }^{2}$ P. G. Bolcatto ${ }^{3}$ C. R. Proetto, ${ }^{4}$ K. Eberl,${ }^{5}$ M. Hauser,${ }^{5}$ and C. Thomsen ${ }^{1}$ \\ ${ }^{1}$ Institut für Festkörperphysik, Technische Universität Berlin, Hardenbergstr. 36, 10623 Berlin, Germany \\ ${ }^{2}$ ICREA Research Professor, Institut de Ciència de Materials de Barcelona, Campus de la UAB, 08193 Bellaterra, Spain \\ ${ }^{3}$ Facultad de Ingeniería Química and Facultad de Humanidades y Ciencias, \\ Universidad Nacional del Litoral, 3000 Santa Fe, Argentina \\ ${ }^{4}$ Centro Atómico Bariloche and Instituto Balseiro, 8400 S. C. de Bariloche, Río Negro, Argentina \\ ${ }^{5}$ MPI für Festkörperforschung, Heisenbergstr. 1, 70569 Stuttgart, Germany
}

(Dated: September 26, 2018)

Recent claims of an experimental demonstration of spontaneous spin polarisation in dilute electron gases [1] revived long standing theoretical discussions [2, 3]. In two dimensions, the stabilisation of a ferromagnetic fluid might be hindered by the occurrence of the metalinsulator transition at low densities [4]. To circumvent localisation in the two-dimensional electron gas (2DEG) we investigated the low populated second electron subband, where the disorder potential is mainly screened by the high density of the first subband. This letter reports on the breakdown of the spin symmetry in a 2DEG, revealed by the abrupt enhancement of the exchange and correlation terms of the Coulomb interaction, as determined from the energies of the collective charge and spin excitations. Inelastic light scattering experiments and calculations within the time-dependent local spin-density approximation give strong evidence for the existence of a ferromagnetic ground state in the diluted regime.

Recently we have shown experimentally as well as theoretically that at low temperatures and zero magnetic field a 2D electron gas realized in a GaAs single quantum well (SQW) undergoes a first-order phase transition, as result of intersubband exchange-correlation terms when the second electron subband becomes populated [5]. The situation of two occupied subbands resembles that of double layer structures, a system with a rich ground state phase diagram, where a collapse of the normal metallic state towards a spontaneous magnetic order at very low densities was also predicted [6, 7]. Evidence of this first order phase transition is the abrupt renormalisation of the second subband energy and its sudden population with electrons [8] as observed by photoluminescence (PL) spectra. The first-order character of the transition was confirmed by self-consistent density-functional calculations, including exchange interactions exactly [9, 10]. The exact-exchange theory predicted also a spontaneous breakdown of the spin symmetry to take place for a low population of the second subband. In this situation a new phase was found in PL spectra at low temperatures and at zero magnetic field [5]. The behaviour of this phase by varying density, temperature and external magnetic field speaks for the formation of spin-polarised domains with different in-plane magnetisation, as expected for planar ferromagnets to minimise the stray field. Moreover, last year possible ferromagnetism was claimed to be observed in transport experiments on a GaAs heterostructure with a single occupied subband [1]. This evidence, though, is not conclusive due to the effects of localisation mentioned above. In this work we investigate the elementary excitations of the 2DEG, observing a strong enhancement of the exchange-correlation contributions occurring at very low population of the second subband. Densityfunctional calculations within the time-dependent local spin-density approximation (TDLSDA) confirm the existence of such spin polarised phase.

The 2DEG forms in modulation-doped GaAs $/ \mathrm{Al}_{0.33} \mathrm{Ga}_{0.67} \mathrm{As}$ single quantum wells of 20 , 25 and $30 \mathrm{~nm}$ well thickness grown by molecular-beam epitaxy. The 2DEG has a mean mobility of about $8 \times 10^{5} \mathrm{~cm}^{2} / \mathrm{Vs}$ and a density such that only the lowest subband is occupied, with Fermi energies $E_{F}$ between 25 and $28 \mathrm{meV}$ at $4.2 \mathrm{~K}$. The electron density can be changed by applying a dc bias between the 2DEG and a metallic back contact. Photoluminescence and inelastic light scattering spectra were obtained using low power densities in the range of 0.5 to $5 \mathrm{~W} / \mathrm{cm}^{2}$. Light scattering spectra were recorded in backscattering geometry using a line focus in order to avoid heating of the 2DEG at the required laser powers.

The set of spectra in Fig. 1 illustrates the evolution of the energy of the charge (CDE), spin (SDE) and singleparticle excitations (SPE), associated with the intersubband transitions $0 \rightarrow 1$ and $1 \rightarrow 2$, when varying the density of the electron gas. The shift of the $\mathrm{SPE}_{01}$ to lower energies and of the $\mathrm{SPE}_{12}$ to higher energies are consequences of the renormalisation of the second subband with increasing occupation. Furthermore, the change in the energy separation between excitations indicates a dependence of the many-body corrections on electron density 12,13 .

As explained in the Appendix, within the formalism of TDLSDA [14] and in the long wavelength limit $(q \approx 0$ for backscattering geometry), the energies of the charge and spin-density excitation $\hbar \omega_{\mu}$ can be written in the following compact way []

$\left(\hbar \omega_{\mu}\right)^{2}-E_{i, i+1}^{2}=2 \delta n_{i, i+1} E_{i, i+1} \gamma_{\mu}^{i, i+1}$,

with $\delta n_{i, i+1}=n_{i}-n_{i+1}$ being the difference in the subband densities and $E_{i, i+1}=E_{i+1}-E_{i}$, where $E_{i}$ are the subband energies. In the paramagnetic $(\mathrm{P})$ phase, the index $\mu=\mathrm{CDE}$, SDE describes the uncoupled charge and spin density excitations. According to standard nota- 


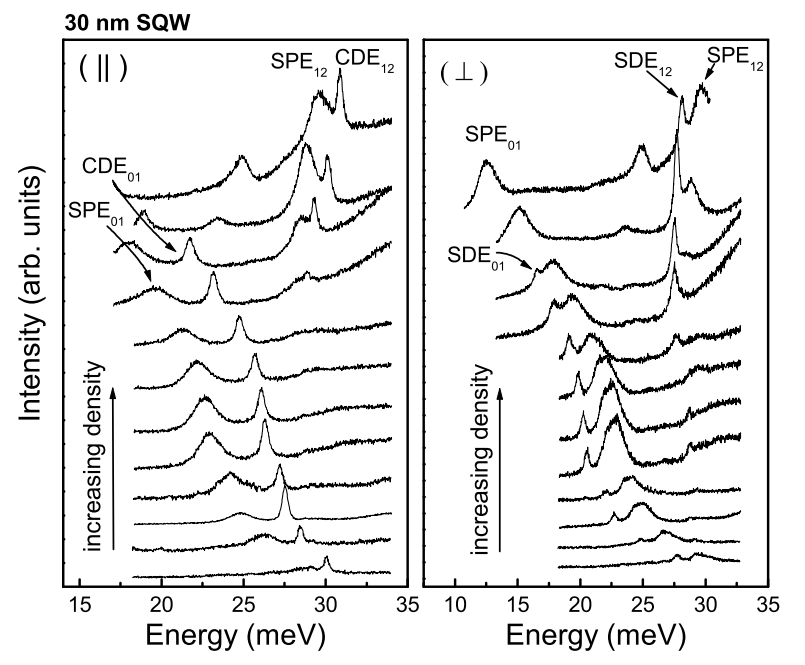

FIG. 1: Inelastic light scattering spectra of a $30 \mathrm{~nm}$ wide SQW obtained at $2 \mathrm{~K}$, with different voltages and in polarised $(\|)$ and depolarised $(\perp)$ configuration. In the spectra with parallel polarisation the CDE associated with intersubband transitions between the first and the second subband $0 \rightarrow 1$, and between the second and the third subbands $1 \rightarrow 2$ are apparent. The corresponding SDE, in contrast, are observed in depolarised configuration. The broader peak that appears in spectra with both polarisations is assigned to the singleparticle excitation SPE and is centred at the intersubband energy $E_{i j}$, with $(i j)$ being (01) or (12).

tions, $\gamma_{C D E}^{i, i+1}=\left(\alpha^{i, i+1} / \tilde{\epsilon}-\beta_{C D E}^{i, i+1}\right)$, and $\gamma_{S D E}^{i, i+1}=-\beta_{S D E}^{i, i+1}$. $\tilde{\epsilon}(\omega)$ is the contribution of the polar lattice to the dielectric function [12]. In the ferromagnetic phase $(\mathrm{F})$, charge and spin are intrinsically linked, thus, there is a single collective mode of mixed character (denoted with subindex $\mu=\mathrm{M}$ ). The depolarisation shift $\alpha$ is a positive Hartree contribution that in the paramagnetic phase typically dominates over the exchange-correlation term $\beta_{C D E}$, whereas $\gamma$ is dominated by $\beta$ in the ferromagnetic case. The explicit expressions for $\alpha, \beta_{C D E}, \beta_{S D E}$, and $\gamma_{M}$ in terms of matrix elements of the Hartree and exchange-correlation potentials are given in the Appendix.

In Fig. 2] we show an unexpected behaviour of the spindensity mode: it becomes softer, i.e. decreases in absolute value when the Fermi level is resonant with the second subband, recovering the normal behaviour at higher densities. From the study of the formation of a non trivial magnetic order, intensively investigated in double-layer systems [15], a softening of the $\mathrm{SDE}_{01}$ can be considered as an indication of ferromagnetism in the second subband. In our system the softening is rather weak, probably due to the large contribution of electrons in the paramagnetic first subband. Comparing the three QW widths (Fig. 2(a)) it can be seen that the SDE softening is enhanced for the narrowest QW. That speaks sensibly for

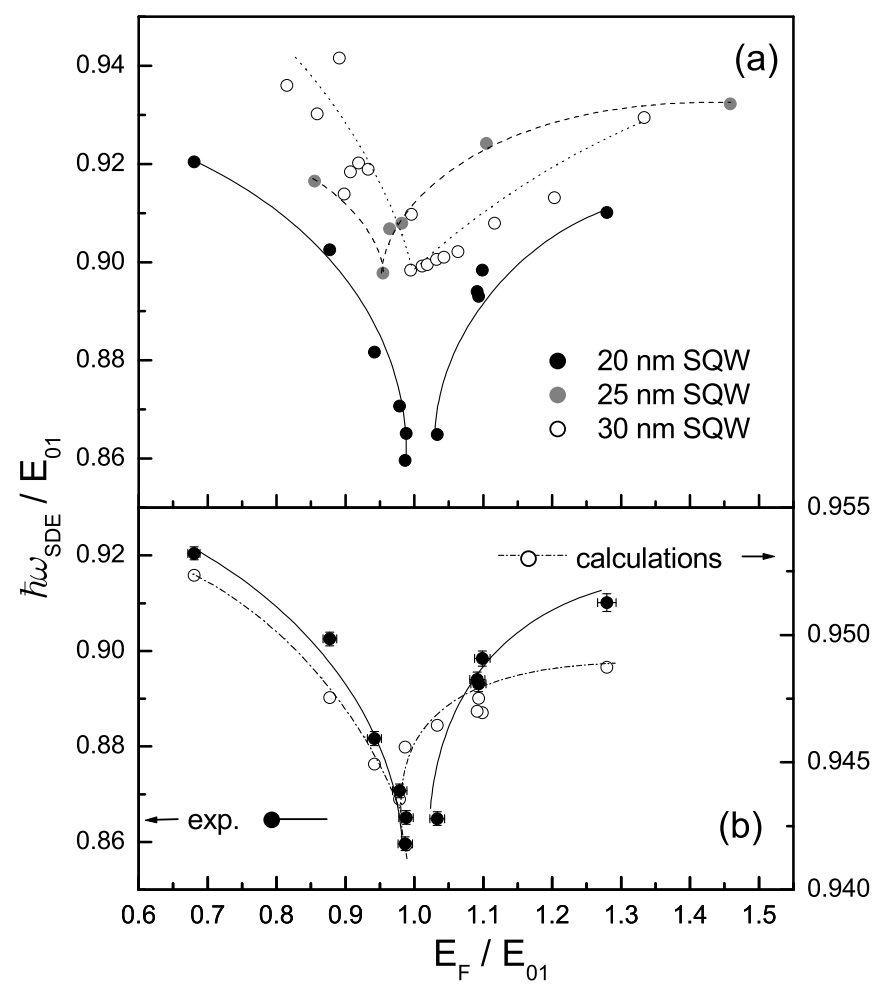

FIG. 2: The $0 \rightarrow 1$ spin-density mode softening as a function of the dimensionless parameter $E_{F} / E_{01}$, being $E_{F} / E_{01}=1$ the borderline between one and two occupied subbands. a) Comparison of the SDE softening among the 20, 25, and 30 $\mathrm{nm}$ wide SQW. b) Comparison of the experimental SDE softening (solid circles, left axis) for the $20 \mathrm{~nm}$ wide SQW with TDLSDA results (open circles, right axis). The lines are guides to the eye.

a dependence of the possible ferromagnetic order on the strength of the wave function confinement. In Fig. 2(b) we compare the experimental data (solid points) with the excitation energies yielded by TDLSDA calculations (empty points). The left (right) axis corresponds to the experimental (calculated) results. The underestimation of the softening resulting from the calculations can be attributed to shortcomings of the TDLSDA in the treatment of exchange and correlation but also to the fact that the calculations always yield a paramagnetic ground state for this high total density.

For this reason and in order to exclude the effect of the electrons in the first paramagnetic subband, we investigate higher intersubband excitations associated with the transition $1 \rightarrow 2$. These excitations occur between the sparsely populated second subband and the empty third subband. Only electrons from the spin polarised phase in $E_{1}$ contribute to the $\mathrm{SDE}_{12}$. In the series of spectra of Fig. 1 the excitations $\mathrm{SPE}_{12}, \mathrm{SDE}_{12}$ and $\mathrm{CDE}_{12}$ become apparent when the second subband is populated. In particular, the $\mathrm{CDE}_{12}$ shows up exactly on top of the $\mathrm{SPE}_{12}$ due to the collapse of the Hartree term $\alpha^{12}$ at very low densities [16]. In contrast, the $\mathrm{SDE}_{12}$ is always shifted 
down from the $\mathrm{SPE}_{12}$. From the measured energies of the excitations obtained with cross polarisation and using Eq. (1) we determine the many-body correction energy $\left(2 n_{1} \gamma^{12}\right)$. Multiplying by $n_{1}$ we avoid divergencies when the density goes to zero. The results are shown in Fig. 3 The striking result of this work is the observation of an

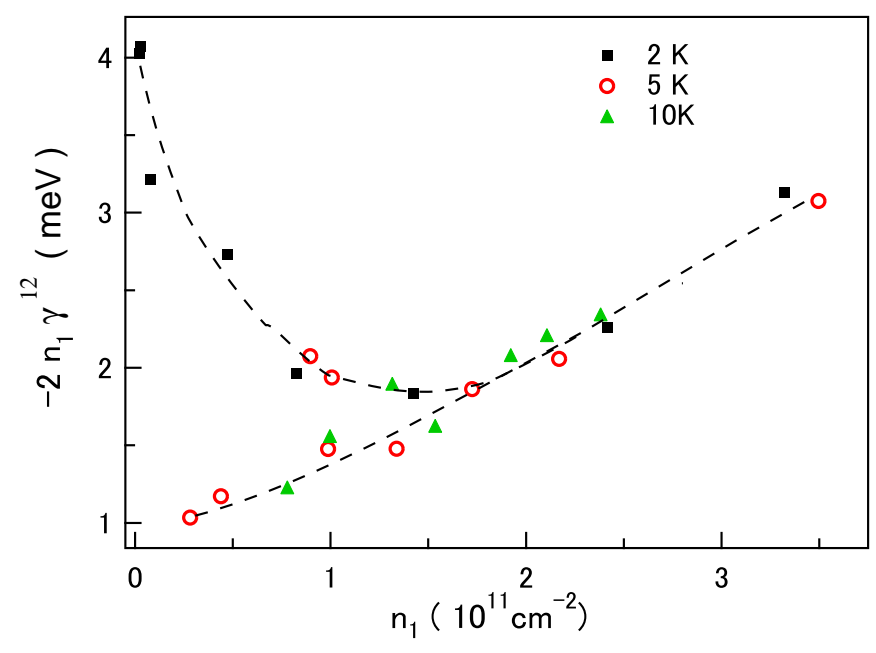

FIG. 3: Enhancement of the exchange-correlation energy contribution $2 n_{1} \gamma^{12}$, as a function of the density $n_{1}$ of the second subband, for the $30 \mathrm{~nm}$ wide SQW and at temperatures $\mathrm{T}=2 \mathrm{~K}$ (circles), $\mathrm{T}=5 \mathrm{~K}$ (squares) and $\mathrm{T}=10 \mathrm{~K}$ (triangles). Curves are a guide to the eye.

enhancement of the exchange-correlation contribution at low temperature when the system enters the region of the spin-instability. This leads to a pronounced softening of the collective mode associated with electronic transitions $1 \rightarrow 2$. The diluted electron gas of the second subband becomes thus unstable against spin-flip excitations, which trigger the transition into the ferromagnetic phase. By increasing the temperature a scaling down of the exchange-correlation energy occurs, in accordance with the disappearance of the new phase observed in the PL spectra, as discussed in previous work [5].

For a better understanding of the physical origin of this transition it is instructive to perform the corresponding calculation of the excitations. To avoid the effect of the high density in the first subband we constructed an auxiliary structure, using for its intersubband energy $E_{01}$ and density $n_{0}$, the experimental values $E_{12}$ and $n_{1}$, respectively. Doing so, the calculations can yield now a ferromagnetic ground state and the corresponding excitations.

Figure 4 compares the experimental $\gamma_{\text {exp }}^{12}$ values, as obtained from the excitations measured in cross polarisation at $2 \mathrm{~K}$, with the calculated ones. TDLSDA predicts the occurrence of a ferromagnetic phase, characterised by $\gamma_{M}$ (open circles), for densities below $0.34 \times 10^{11} \mathrm{~cm}^{-2}$ and a paramagnetic phase otherwise, characterised by the excitonic shifts $\beta_{C D E}$ and $\beta_{S D E}$ (triangles). Inspection of the different terms in $\gamma_{M}$ reveals that its strong

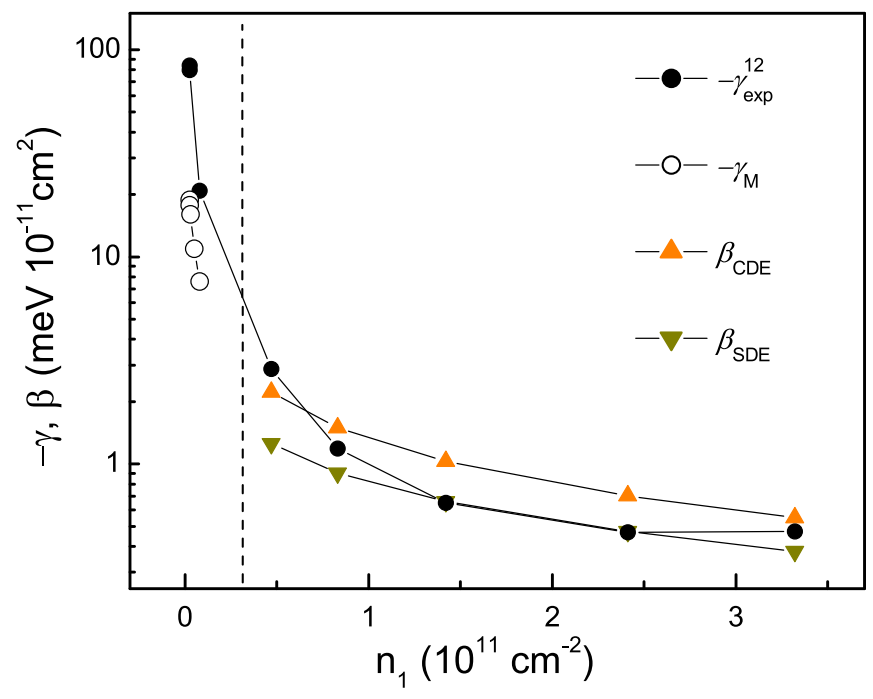

FIG. 4: Comparison between the calculated many-body corrections $\gamma_{M}$ (ferromagnetic solution), $\beta_{C D E}$ and $\beta_{S D E}$ (paramagnetic solution), obtained within TDLSDA and $\gamma_{\text {exp }}^{12}$ from depolarised spectra at $2 \mathrm{~K}$ of the $30 \mathrm{~nm}$ wide SQW. The dashed line divides the ferromagnetic (left) from the paramagnetic (right) region.

increase at low densities is mainly due to the fact that the contribution from correlation has the opposite sign than the one to $\beta_{S D E}$ in the paramagnetic phase. Thus, correlation adds to the effect of exchange in the spinpolarised case, which favours ferromagnetism. In fact, the calculated values of $\beta_{S D E}$ and $\gamma_{M}$ at high and low densities, respectively, agree well with $\gamma_{\text {exp }}^{12}$. The range of stability of the ferromagnetic solution is also in nice agreement with the data of Fig. 3, where the strong enhancement of exchange-correlation vertex corrections, observed at low temperatures, occurs at densities below $1 \times 10^{11} \mathrm{~cm}^{-2}$. In this way, we have obtained from light scattering experiments and local spin-density calculations the first compelling evidence for the existence of a ferromagnetic ground state of 2DEGs in an effectively diluted regime; one of the exciting puzzles of many-body physics stated more than fifty years ago by F. Bloch [3]

\section{APPENDIX A: METHODS}

The ground-state calculations have been performed in the local spin-density approximation (LSDA) in the framework of density-functional theory. We adjusted the geometry of the SQW in order to reproduce the Fermi energy $E_{F}$, and the two subbands energies $E_{0}$ and $E_{1}$ as obtained from photoluminescence experiments.

The energy of the collective excitations of charge (CDE) and spin (SDE) were obtained with TDLSDA from the zeros of the secular determinant of the spindependent dielectric tensor ( $\operatorname{det} \epsilon=0$ ), which can be written in terms of the Hartree and exchange-correlation 
potentials in the subband representation [7]. The corresponding potential matrix elements, as a function of the wavevector $q$ of the excitations, are

$$
\begin{aligned}
\left(V_{H}\right)_{i j, i^{\prime} j^{\prime}}^{\sigma, \sigma^{\prime}}(q) & =\frac{1}{A} \int d z \int d z^{\prime}\left(2 \pi e^{2} / \varepsilon q\right) \exp \left(-q\left|z-z^{\prime}\right|\right) \\
& \times \phi_{i \sigma}^{*}(z) \phi_{j \sigma}(z) \phi_{i^{\prime} \sigma^{\prime}}^{*}\left(z^{\prime}\right) \phi_{j^{\prime} \sigma^{\prime}}\left(z^{\prime}\right), \quad(\mathrm{A} 1) \\
\left(V_{x c}\right)_{i j, i^{\prime} j^{\prime}}^{\sigma, \sigma^{\prime}}(q) & =\frac{1}{A} \int d z \phi_{i \sigma}^{*}(z) \phi_{j \sigma}(z) \phi_{i^{\prime} \sigma^{\prime}}^{*}(z) \phi_{j^{\prime} \sigma^{\prime}}(z) \\
& \times\left[K_{x c}(z)+\left(\sigma+\sigma^{\prime}\right) J_{x c}(z)+\sigma \sigma^{\prime} I_{x c}(z)\right] .
\end{aligned}
$$

$\phi_{i \sigma}(z)$ are the self-consistent wave functions that diagonalise the effective one-dimensional LSDA Hamiltonian, after assuming translational invariance along the $(x, y)$ plane $(\operatorname{area} A) . i, j, i^{\prime}, j^{\prime}$ are subband indexes, and $\sigma, \sigma^{\prime}=1(-1)$ for spin $\uparrow(\downarrow)$. Eq. A1 corresponds to the Hartree contribution, with $\varepsilon$ being the dielectric constant of GaAs. Eq. A2 corresponds to the exchangecorrelation contribution, with $K, J$, and $I$ being derivatives of the exchange-correlation energy functional with respect to the density and magnetisation of the 2DEG 7]. Making a two-subband approximation $\left(i=i^{\prime}, j=j^{\prime}\right)$, and in the long wavelength limit $(q \rightarrow 0)$, the eigenmode equation $\operatorname{det} \epsilon=0$ can be solved analytically for $(\hbar \omega)^{2}$. The resulting equations for the $\mathrm{P}$ phase take the form of Eq. (1), provided the following identification is made: $\alpha=V_{H}, \beta_{C D E}=-\left(V_{x c}^{\uparrow \uparrow}+V_{x c}^{\uparrow \downarrow}\right) / 2$, and $\beta_{S D E}=-\left(V_{x c}^{\uparrow \uparrow}-V_{x c}^{\uparrow \downarrow}\right) / 2$. For the sake of simplicity, we have eliminated subband indexes, and we have used the symmetry $V_{H}=V_{H}^{\uparrow \uparrow}=V_{H}^{\downarrow \downarrow}=V_{H}^{\uparrow \downarrow}=V_{H}^{\downarrow \uparrow}$. In the $\mathrm{F}$ phase, $\gamma_{M}=\left(V_{H}^{\uparrow \uparrow}+V_{x c}^{\uparrow \uparrow}\right)$. This single collective mode has a mixed CDE and SDE character, and accordingly it has both Hartree and exchange-correlation contributions, the latter being the dominant term in the $\mathrm{F}$ phase.

\section{ACKNOWLEDGMENTS}

We thank W. Dietsche for assistance with the sample design and growth. P. G. acknowledges financial support from the DAAD and the "Berliner Programm zur Förderung der Chancengleichheit". This work is supported in part by the DFG in the framework of Sfb 296. P. G. B. acknowledges to U.N.L., ANPCyT and Fundación Antorchas by Grants PE-213, 03-11736 and 14248-128 respectively. C. R. P. and P. G. B. are partially supported by CONICET.
[1] D. P. Young, D. Hall, M. D. Torelli, Z. Fisk, J. L. Sarrao, J. D. Thompson, H.-R. Ott, S. B. Oseroff, R. G. Goodrich, and R. Zysler, "High-temperature weak ferromagnetism in a low-density free-electron gas", Nature 397, 412 (1999).

[2] D. M. Ceperley, "Return of the itinerant electron", Nature 397, 386 (1999).

[3] F. Bloch, "Bemerkungen der Elektronentheorie des Ferromagnetismus und der elektrische Leitfähigkeit", Z. Phys. 57, 545 (1929).

[4] E. Abraham, P. W. Anderson, D. C. Licciardello, and T. V. Ramakrishnan, "Scaling Theory of Localization: Absence of Quantum Diffusion in Two Dimensions", Phys. Rev. Lett. 42, 673 (1979).

[5] A. R. Goñi, P. Giudici, F. A. Reboredo, C. R. Proetto, C. Thomsen, K. Eberl, and M. Hauser, "Evidence of spontaneous spin polarisation in the two-dimensional electron gas" Phys. Rev. B 70, 195331 (2004).

[6] F. A. Reboredo and C. R. Proetto, "Novel Magnetic Instabilities in Semiconductor Double Quantum Wells" Phys. Rev. Lett. 79, 000463 (1997)

[7] P. G. Bolcatto, C. R. Proetto, and F. A. Reboredo, "Collective excitations in spontaneously spin-polarized phases of semiconductor double-quantum-well systems", Phys. Rev. B 67, 073304 (2003).

[8] A. R. Goñi, U. Haboeck, C. Thomsen, K. Eberl, F. A. Reboredo, C. R. Proetto, and F. Guinea, "Exchange instability of the two-dimensional electron gas in semiconductor quantum wells", Phys. Rev. B 65, R121313 (2002).

[9] F. A. Reboredo and C. R. Proetto, "Exact-exchange den- sity functional theory for quasi-two-dimensional electron gases", Phys. Rev. B 67, 115325 (2003).

[10] S. Rigamonti, F.A. Reboredo, and C.R. Proetto, "Exactexchange density-functional theory applied to a strongly inhomogeneous electron gas", Phys. Rev. B 68, 235309 (2003).

[11] A. Ghosh, C. J. B. Ford, M. Pepper, H. E. Beere and D. A. Ritchie, "Possible Evidence of a Spontaneous Spin Polarization in Mesoscopic Two-Dimensional Electron System, Phys. Rev. Lett. 92, 116601 (2004).

[12] A. Pinczuk, S. Schmitt-Rink, G. Danan, J. P. Valladares, L. N. Pfeiffer, and K. W. West, "Large exchange interactions in the electron gas of GaAs quantum wells", Phys. Rev. Lett. 63, 1633 (1989).

[13] D. Gammon, B. V. Shanabrook, J. C. Ryan, and D. S. Katzer, "Spin-density waves in a quasi-two-dimensional electron gas", Phys. Rev. B 41, 12311 (1990);

[14] W. Kohn and P. Vahistha, in Theory of the Inhomogeneous Electron Gas, edited by S. Ludnqvist and N. H. March (Plenum, New York, 1983); W. Kohn, Rev. Mod. Phys. 71, 1253 (1999).

[15] P. G. Bolcatto and C. R. Proetto, "Spin-Density and Charge-Density Excitations in the Paramagnetic Phase of Semiconductor Double Quantum Well Systems", Phys. Rev. Lett. 85, 1734 (2000).

[16] S. Ernst, A. R. Goñi, K. Syassen, and K. Eberl, "Collapse of the Hartree term of the Coulomb interaction in a very dilute 2D electron gas", Phys. Rev. Lett. 72, 4029 (1994). 